\title{
Dynamics of a stage-structured single population model with state-dependent delay
}

\author{
Yan Wang ${ }^{1}$, Xianning Liu ${ }^{1 *}$ (D) and Yangjiang Wei ${ }^{2}$
}

\author{
"Correspondence: \\ liuxn@swu.edu.cn \\ ${ }^{1}$ Key Laboratory of \\ Eco-environments in Three Gorges \\ Reservoir Region (Ministry of \\ Education), School of Mathematics \\ and Statistics, Southwest University, \\ Chongqing, China \\ Full list of author information is \\ available at the end of the article
}

\begin{abstract}
In this paper, a novel stage-structured single population model with state-dependent maturity delay is formulated and analyzed. The delay is related to the size of population and taken as a non-decreasing differentiable bounded function. The model is quite different from previous state-dependent delay models in the sense that a correction term, $1-\tau^{\prime}(z(t)) \dot{z}(t)$, is included in the maturity rate. Firstly, positivity and boundedness of solutions are proved without additional conditions. Secondly, existence of all equilibria and uniqueness of a positive equilibrium are discussed. Thirdly, local stabilities of the equilibria are obtained. Finally, permanence of the system is analyzed, and explicit bounds for the eventual behaviors of the immature and mature populations are established.
\end{abstract}

MSC: Primary 92D25; secondary 34K20

Keywords: Stage structure; State-dependent delay; Correction term; Stability; Permanence

\section{Introduction}

In a natural ecosystem, the individual members of the population have a life history that takes them through two or more stages, especially, with regard to mammalian populations, which usually exhibit two distinct stages: immature and mature stages [1-4]. What is more, the delay arises frequently as the maturation time from birth to adulthood under the background of population dynamics, and there are different types of the maturity time delay, such as discrete, distributed, stochastic, time-dependent and state-dependent delays, etc. [4-11].

The authors of [12] considered and analyzed a growth model of blowflies with constant maturity time delay. According to the data of blowflies growth experiments in [13], they presented some numerical simulations to justify the theoretical analysis. Thus, it is more practical to consider time delays and stage structures in the study of a population model.

In 1990, the authors of [14] developed and analyzed the following stage structure model of population growth with a constant maturity time delay:

$$
\left\{\begin{array}{l}
\frac{d x(t)}{d t}=\alpha y(t)-\gamma x(t)-\alpha y(t-\tau) e^{-\gamma \tau}, \\
\frac{d y(t)}{d t}=\alpha y(t-\tau) e^{-\gamma \tau}-\beta y^{2}(t),
\end{array}\right.
$$

(c) The Author(s) 2018. This article is distributed under the terms of the Creative Commons Attribution 4.0 International License (http://creativecommons.org/licenses/by/4.0/), which permits unrestricted use, distribution, and reproduction in any medium, provided you give appropriate credit to the original author(s) and the source, provide a link to the Creative Commons license, and indicate if changes were made. 
where $x(t)$ and $y(t)$ represent the immature and mature population densities, respectively; the time delay $\tau$ represents the time from birth to maturity; parameters $\alpha$ and $\gamma$ represent the birth and death rates of the immature population, respectively; $\beta$ represents the mature death and overcrowding rate.

Before World War II, people discovered that juvenile seals needed five years to mature, small whales needed seven to ten years, and big whales needed 12 to 15 years. After the war, owing to the introduction of factory ships and capture of a large number of whales, the number of the krill available for the seals and the remaining whales had a significant increase. An interesting phenomenon was then observed that the maturity time of seals and whales has shortened. Seals needed three to four years and small whales only took five years. Additionally, there was a significant reduction of maturity time for large whales [15]. Therefore, for Antarctic whale and seal populations, their maturity time is a function of the number of krill. Furthermore, it is quite natural to suppose that the number of krill available in a closed environment is a function of the total population.

This suggests that due to the environmental complexity, however, the time delay may be constantly adjusted as the state changes, that is, the time delay is state-dependent and the constant time delay is no longer reasonable (see [16-19]). In view of this, on the basis of model (1.1), the authors of [5] changed the constant delay $\tau$ into a state-dependent delay $\tau(z(t))$ to formulate the following state-dependent delay model:

$$
\left\{\begin{array}{l}
\frac{d x(t)}{d t}=\alpha y(t)-\gamma x(t)-\alpha y(t-\tau(z(t))) e^{-\gamma \tau(z(t))}, \\
\frac{d y(t)}{d t}=\alpha y(t-\tau(z(t))) e^{-\gamma \tau(z(t))}-\beta y^{2}(t),
\end{array}\right.
$$

where the state-dependent time delay $\tau(z(t))$ is taken to be an increasing differentiable bounded function of the total population $z(t)=x(t)+y(t)$.

Since then, more and more state-dependent delay differential equations were applied to describe the dynamical behaviors of stage-structured models originated from population growth [19-25].

In 2004, based on model (1.2), the authors of [26] studied the following stage-dependent population model with a state-dependent time delay:

$$
\left\{\begin{array}{l}
\frac{d x(t)}{d t}=R(y(t))-\gamma x(t)-R(y(t-\tau(z(t)))) e^{-\gamma \tau(z(t))}, \\
\frac{d y(t)}{d t}=R\left(y(t-\tau(z(t))) e^{-\gamma \tau(z(t))}-\beta y(t),\right.
\end{array}\right.
$$

where the immature birth rate $R(y(t))$ is taken as a general function of the present mature population and the death rate for the mature one is a constant.

Subsequently, [27] and [28] investigated a two cooperative mature species system with a state-dependent delay and a cooperative model composed of two species with a stage structure and state-dependent maturity delays, respectively.

However, it is obvious that those state-dependent delay equations are all directly replacing constant delays in classical models of the form

$$
\frac{d X(t)}{d t}=F(X(t-\tau), X(t)), \quad \tau>0
$$


by state-dependent delays, hence one obtains the following equations:

$$
\frac{d X(t)}{d t}=F(X(t-\tau(X(t))), X(t)) .
$$

Is this approach appropriate and in line with population modeling? Since if the maturity delay is not constant but state-dependent, then changes in the number of mature individuals do not only depend on reproduction and death, but also on the changing definition of maturity, that is, changes of $\tau(X(t)))$. As we shall show later, the correct extension of model (1.2) in terms of a state-dependent mature delay involves a correction term, $1-\tau^{\prime}(z(t)) \dot{z}(t)$, which takes into account the aforesaid changes.

Moreover, Macdonald [29] indicated that one must begin with an age structured model to incorporate maturation data in a model, which is indispensably formulated in terms of partial differential equations. This way it is guaranteed that the model can properly be substituted by that formulated according to a functional differential equation.

Our paper is organized as follows. In Sect. 2, we formulate a novel specie stagestructured model with a state-dependent maturation delay. In Sect. 3, we discuss the positivity and boundedness of solutions, as well as the existence of all equilibria and uniqueness of a positive equilibrium. In Sect. 4, we analyze the linear stability of equilibria. In Sect. 5, we discuss the permanence of the system. Finally, in Sect. 6, we briefly discuss and summarize our results.

\section{Model formulation and hypotheses}

\subsection{Model formulation}

Motivated by $[8,29,30]$, we begin with a two-stage population model (immature and mature stages). In order to distinguish immature individuals, $x(t)$, from mature ones, $y(t)$, we introduce a threshold age $\tau(z(t))$, which is the maturation time for an immature individual that matures at time $t$ depending on the total population $z(t)=x(t)+y(t)$. Let $\rho(t, a)$ be the density of population of age $a$ at time $t$. Then the number of immature individuals, $x(t)$, and mature individuals, $y(t)$, respectively, are given by

$$
x(t)=\int_{0}^{\tau(z(t))} \rho(t, a) d a \quad \text { and } \quad y(t)=\int_{\tau(z(t))}^{\infty} \rho(t, a) d a
$$

The evolution of population is represented by the following age structure partial differential equations [31, 32]:

$$
\begin{aligned}
& \frac{\partial \rho(t, a)}{\partial t}+\frac{\partial \rho(t, a)}{\partial a}=-\gamma \rho(t, a), \quad \text { if } a \leq \tau(z(t)), \\
& \frac{\partial \rho(t, a)}{\partial t}+\frac{\partial \rho(t, a)}{\partial a}=-\beta y(t) \rho(t, a), \quad \text { if } a>\tau(z(t)),
\end{aligned}
$$

where each individual from $x(t)$ dies at a constant rate $\gamma$ and that from $y(t)$ at a nonconstant rate $\beta y(t)$.

Taking the derivatives of $x(t)$ and $y(t)$, respectively, and combining with (2.1), we get

$$
\begin{aligned}
& \frac{d x(t)}{d t}=\rho(t, 0)-\gamma x(t)-\left[1-\tau^{\prime}(z(t)) \dot{z}(t)\right] \rho(t, \tau(z(t))), \\
& \frac{d y(t)}{d t}=\left[1-\tau^{\prime}(z(t)) \dot{z}(t)\right] \rho(t, \tau(z(t)))-\rho(t, \infty)-\beta y^{2}(t) .
\end{aligned}
$$


It is necessary to note that a prime refers to differentiation with respect to $z$, and a dot indicates differentiation with respect to time $t$, namely, $\dot{\tau}(z(t))=d \tau(z(t)) / d t=\tau^{\prime}(z(t)) \dot{z}(t)$.

Because any individual cannot live forever, $\rho(t, \infty)$ is regarded as zero. We assume that the immature population produces at a constant rate $\alpha$, so the term $\rho(t, 0)=\alpha y(t)$ represents the number of immature individuals born at time $t$. Therefore, for $t \geq \tilde{\tau}=$ $\max \{\tau(z(t))\}$, we obtain

$$
\rho(t, \tau(z(t)))=\rho(t-\tau(z(t)), 0)=\alpha y(t-\tau(z(t))) e^{-\gamma \tau(z(t))} .
$$

As a result, we have the following stage-structured population model with a statedependent delay:

$$
\left\{\begin{array}{l}
\frac{d x(t)}{d t}=\alpha y(t)-\gamma x(t)-\alpha\left[1-\tau^{\prime}(z(t)) \dot{z}(t)\right] y(t-\tau(z(t))) e^{-\gamma \tau(z(t))}, \\
\frac{d y(t)}{d t}=\alpha\left[1-\tau^{\prime}(z(t)) \dot{z}(t)\right] y(t-\tau(z(t))) e^{-\gamma \tau(z(t))}-\beta y^{2}(t) .
\end{array}\right.
$$

Model (2.2) is clearly different from the previous state-dependent delay equations in the sense that it includes the correction term $1-\tau^{\prime}(z(t)) \dot{z}(t)$ in the maturity rate.

As in $[5,26,28,30]$, to have the possibility of mature individuals becoming immature only by birth, one needs some conditions ensuring that $t-\tau(z(t))$ is a strictly increasing function of $t$. As a matter of fact, from a biological point of view, it is natural that $t-\tau(z(t))$ is a strictly increasing function of $t$. Assume that $r(\xi)$ is the developmental proportion at time $\xi$. Then, when an immature individual moves to the mature state from $t-\tau(z(t))$ to $t$, the cumulative rate of development $r$ should be equal to one, namely,

$$
1=\int_{t-\tau(z(t))}^{t} r(\xi) d \xi
$$

Taking the derivative with respect to $t$, we obtain

$$
1-\tau^{\prime}(z(t)) \dot{z}(t)=\frac{r(t)}{r(t-\tau(z(t)))}>0 .
$$

It implies that $t-\tau(z(t))$ is a strictly increasing function of $t$ and the maturity time delay $\tau(z(t))$ doesn't change arbitrarily over time.

\subsection{Model hypotheses}

The hypotheses for model (2.2) are as follows:

(A1) Parameters $\alpha, \gamma, \beta$ are all positive constants;

(A2) The state-dependent maturity time delay $\tau(z)$ is an increasing differentiable bounded function of the total population $z=x+y$, where $\tau^{\prime}(z) \geq 0, \tau^{\prime \prime}(z) \leq 0$, and $0<\tau_{m} \leq \tau(z) \leq \tau_{M}$ with $\tau(0)=\tau_{m}$ and $\tau(+\infty)=\tau_{M}$.

Throughout this paper, we will do qualitative analysis for system (2.2). The initial conditions for system (2.2) are

$$
\begin{aligned}
& y(s)=\Phi(s) \geq 0, \\
& x(s)=\Psi(s) \geq 0 \quad \text { for all } s \in\left[-\tau_{M}, 0\right],
\end{aligned}
$$


with

$$
\Psi(0)=\int_{-\tau(z(0))}^{0} \alpha \Phi(s) e^{\gamma s} d s
$$

which denotes the size of the immature population surviving to time $t=0$, where $\tau(z(0))$ is the maturation time at $t=0$, namely,

$$
\tau(z(0))=\tau\left(\Phi(0)+\int_{-\tau(z(0))}^{0} \alpha \Phi(s) e^{\gamma s} d s\right)
$$

\section{Preliminary results}

In this section, we will discuss the positivity and boundedness of solutions and the existence of all equilibria and uniqueness of a positive equilibrium.

\subsection{Positivity and boundedness}

Theorem 3.1 Let $\Phi(t) \geq 0, \Psi(t) \geq 0$ for $-\tau_{M} \leq t \leq 0$ and $\Phi(0)>0$. Then $y(t)>0$ and $x(t)>0$ for all $t \geq 0$.

Proof Since $1-\tau^{\prime}(z(t)) \dot{z}(t)>0$, for $-\tau_{M} \leq t \leq 0$, we obtain

$$
\dot{y}(t) \geq-\beta y^{2}(t)
$$

By comparison, $y(t) \geq y_{1}(t)$ where $y_{1}(t)$ is the solution of $\dot{y}_{1}(t)=-\beta y_{1}^{2}(t), y_{1}(0)=\Phi(0)>0$. Therefore, $y(t)>0$ for all $-\tau_{M} \leq t \leq 0$. We can continue the argument to all positive times, so $y(t)>0$ for all $t \geq 0$. Now let us prove the positivity of $x(t)$. Integrating the first equation of (2.2), we get

$$
\begin{aligned}
x(t) & =e^{-\gamma t}\left(\Psi(0)+\int_{0}^{t} \alpha y(s) e^{\gamma s} d s-\int_{-\tau(z(0))}^{t-\tau(z(t))} \alpha y(s) e^{\gamma s} d s\right) \\
& =e^{-\gamma t}\left(\int_{-\tau(z(0))}^{0} \alpha y(s) e^{\gamma s} d s+\int_{0}^{t} \alpha y(s) e^{\gamma s} d s-\int_{-\tau(z(0))}^{t-\tau(z(t))} \alpha y(s) e^{\gamma s} d s\right) \\
& =\int_{t-\tau(z(t))}^{t} \alpha y(s) e^{\gamma s} d s .
\end{aligned}
$$

By the positivity of $y(t)$ and $\tau(z(t))$, we have $x(t)>0$. The proof is complete.

Remark 3.1 In Theorem 3.5 of [5] and Theorem 2.6 of [28], one needs the following stringent conditions for $\tau^{\prime}(z(t))$ to ensure the positivity of $x$ :

(i) $\tau^{\prime}(z)<4 \beta / \alpha^{2}$;

(ii) $\tau^{\prime}(z)>0$ is small enough so that the inequality

$$
K_{m} \int_{t-\tau_{m}}^{t} e^{\gamma s} d s>\Delta \int_{-\tau}^{t-\tau_{m}} \frac{\alpha^{2} \tau^{\prime}(z)}{4 \beta-\alpha^{2} \tau^{\prime}(z)} e^{\gamma s} d s,
$$

holds for all values of t, where $K_{m}>0$ and $y(t)>K_{m}$ for all $t \geq 0$.

As a matter of fact, according to Theorem 3.1, we see that neither of these conditions is necessary. 
Theorem 3.2 Let $\Phi(t) \geq 0, \Psi(t) \geq 0$ for $-\tau_{M} \leq t \leq 0$ and $\Phi(0)>0$, then the solution $(x(t), y(t))$ of system (2.2) is uniformly ultimately bounded for $t \geq 0$.

Proof Define a Lyapunov function as follows:

$$
V(t)=x(t)+y(t)
$$

Calculating the time derivative of $V(t)$ along the solutions of system (2.2), we obtain

$$
\begin{aligned}
\dot{V}(t) & =\dot{x}(t)+\dot{y}(t) \\
& =\alpha y(t)-\gamma x(t)-\beta y^{2}(t) \\
& =-\gamma V(t)+(\alpha+\gamma) y(t)-\beta y^{2}(t) \\
& \leq-\gamma V(t)+M,
\end{aligned}
$$

where $M>0$ is the maximum of the quadratic function $(\alpha+\gamma) y(t)-\beta y^{2}(t)$. Therefore, $\lim \sup _{t \rightarrow \infty} V(t) \leq M / \gamma$ and the solution of system (2.2) is uniformly ultimately bounded.

Obviously, it implies that $z(t)$ is bounded, which will be used in the proof of Theorem 5.1. In addition, $x(t) \leq M / \gamma$ and $y(t) \leq M / \gamma$, which is the ultimate upper bound for all the solutions. For the upper bound of each solution, we have the following results.

Theorem 3.3 Let $\Phi(t) \geq 0$ for $-\tau_{M} \leq t \leq 0$ and $\Phi(0)>0$. Then there exists $a \Delta=\Delta(\Phi)>0$ such that $y(t) \leq \triangle$ for $t \geq 0$, where $\triangle=\max \left\{\sup _{-\tau_{M} \leq t \leq 0} \Phi(t), \alpha \beta^{-1} e^{-\gamma \tau_{m}}\right\}$.

Proof Our proof is divided into three cases.

Case (a). Firstly, we start with the case where both $y(t)$ and $z(t)$ are eventually monotonic. If $z(t)$ is eventually decreasing, since $z(t)=x(t)+y(t)$, then $y(t)$ is bounded. Suppose that both $y(t)$ and $z(t)$ are eventually increasing, i.e., $\dot{y}(t) \geq 0$ and $\dot{z}(t) \geq 0$ for all $t>T$ for some $T \geq 0$. Then for $t>T+\tau_{M}$

$$
\begin{aligned}
\dot{y}(t) & =\alpha\left[1-\tau^{\prime}(z(t)) \dot{z}(t)\right] y(t-\tau(z(t))) e^{-\gamma \tau(z(t))}-\beta y^{2}(t) \\
& \leq \alpha\left[1-\tau^{\prime}(z(t)) \dot{z}(t)\right] y(t) e^{-\gamma \tau(z(t))}-\beta y^{2}(t) \\
& \leq \alpha y(t) e^{-\gamma \tau(z(t))}-\beta y^{2}(t),
\end{aligned}
$$

since $y(t-\tau(z(t))) \leq y(t)$. This implies that for $t>T$, we have

$$
\begin{aligned}
y(t) & \leq \alpha \beta^{-1}\left[1-\tau^{\prime}(z(t)) \dot{z}(t)\right] e^{-\gamma \tau(z(t))} \\
& \leq \alpha \beta^{-1} e^{-\gamma \tau_{m}} .
\end{aligned}
$$

Case (b). Next, we assume that both $y(t)$ and $z(t)$ are oscillatory. Suppose that there are two sequences $\left\{t_{n}\right\}_{n=1}^{\infty}$ and $\left\{s_{m}\right\}_{m=1}^{\infty}$ such that $\dot{y}\left(t_{n}\right)=0$ and $\dot{z}\left(s_{m}\right)=0, y\left(t_{n}\right)$ and $z\left(s_{m}\right)$ are local maxima of $y(t)$ and $z(t)$, respectively, and that for all $0<t<t_{n}$ and $0<t<s_{m}$, where $m, n \in N$, we have $y(t) \leq y\left(t_{n}\right)$ and $z(t) \leq z\left(s_{m}\right)$. For any given $t_{n}$, let $t_{m}=\max \left\{t_{n} \mid t_{n} \leq t_{m}\right\}$. 
If $t_{m}=s_{m}$, then by using similar methods as in the case (a), we see that $y(t) \leq \alpha \beta^{-1} e^{-\gamma \tau_{m}}$ for all $t<t_{m}$.

If $t_{m}<s_{m}$ and $y\left(t_{m}\right) \leq y\left(s_{m}\right)$, then $\dot{y}\left(s_{m}\right)>0$ for all $t<t_{m}$. Otherwise, there exists a $t \in$ $\left(t_{m}, s_{m}\right)$ such that $\dot{y}(t)=0$, which contradicts the definition of $t_{m}$. Therefore, we obtain

$$
\begin{aligned}
\dot{y}\left(s_{m}\right) & =\alpha\left[1-\tau^{\prime}\left(z\left(s_{m}\right)\right) \dot{z}\left(s_{m}\right)\right] y\left(s_{m}-\tau\left(z\left(s_{m}\right)\right)\right) e^{-\gamma \tau\left(z\left(s_{m}\right)\right)}-\beta y^{2}\left(s_{m}\right) \\
& \leq \alpha y\left(s_{m}\right) e^{-\gamma \tau_{m}}-\beta y^{2}\left(s_{m}\right) .
\end{aligned}
$$

This implies that $y(t) \leq \alpha \beta^{-1} e^{-\gamma \tau_{m}}$.

If $t_{m}<s_{m}$ and $y\left(s_{m}\right) \leq y\left(t_{m}\right)$, then it is clear that $y(t)$ is bounded above.

Case (c). We discuss the case where one of $y(t)$ and $z(t)$ is oscillatory and the other is eventually monotonic. Without loss of generality, suppose that $y(t)$ is oscillatory and $z(t)$ is eventually increasing since the other cases can be worked out similarly. Therefore, there is a sequence $\left\{t_{n}\right\}_{n=1}^{\infty}$ such that $\dot{y}\left(t_{n}\right)=0, y\left(t_{n}\right)$ is a local maximum of $y(t)$, and $y(t) \leq y\left(t_{n}\right)$ for all $0<t<t_{n}$. For the same sequence $\left\{t_{n}\right\}_{n=1}^{\infty}$ presented above, there exists an $N>0$ such that $\dot{z}\left(t_{n}\right) \geq 0$ from the eventual monotonicity of $z(t)$, and thus $y(t)$ is bounded above.

Therefore, choosing $\triangle(\Phi)=\max \left\{\sup _{-\tau_{M} \leq t \leq 0} \Phi(t), \alpha \beta^{-1} e^{-\gamma \tau_{m}}\right\}$, the proof is complete.

Theorem 3.4 Let $\Phi(t)>0$ for $-\tau_{M} \leq t \leq 0$. Then there exists a $\Theta(\Phi)=\Psi(0)+\alpha \gamma^{-1} \Delta$ such that $x(t) \leq \Theta$ for all $t \geq 0$.

Proof Firstly, $\Theta$ is a functional depending only on $\Phi(t)$, since $\Psi(0)=\int_{-\tau_{s}}^{0} \alpha \Phi(s) e^{\gamma s} d s$. Then from system (2.2),

$$
\frac{d x(t)}{d t}=\alpha y(t)-\gamma x(t)-\alpha\left[1-\tau^{\prime}(z(t)) \dot{z}(t)\right] y(t-\tau(z(t))) e^{-\gamma \tau(z(t))} .
$$

Integrating this expression, for $t>0$, we get

$$
\begin{aligned}
x(t) & =e^{-\gamma t} \Psi(0)+\alpha e^{-\gamma t} \int_{0}^{t} e^{r s}\left[y(s)-\left(1-\tau^{\prime}(z) \dot{z}(s)\right) e^{-\gamma \tau(z(s))} y(s-\tau(z(s)))\right] d s \\
& \leq e^{-\gamma t} \Psi(0)+\alpha e^{-\gamma t} \int_{0}^{t} e^{\gamma s} y(s) d s \\
& \leq e^{-\gamma t} \Psi(0)+\alpha e^{-\gamma t} \int_{0}^{t} e^{\gamma s} \Delta d s \\
& =e^{-\gamma t} \Psi(0)+\alpha \gamma^{-1} \Delta\left(1-e^{-\gamma t}\right)<\Psi(0)+\alpha \gamma^{-1} \Delta .
\end{aligned}
$$

Thus, choosing $\Theta(\Phi)=\Psi(0)+\alpha \gamma^{-1} \Delta$, we complete the proof.

\subsection{Existence and patterns of equilibria}

The purpose of this section is to investigate the existence and patterns of equilibria $(x, y)$ of system (2.2), which satisfy

$$
\left\{\begin{array}{l}
\alpha y-\gamma x-\alpha\left[1-\tau^{\prime}(z) \dot{z}\right] y e^{-\gamma \tau(z)}=0, \\
\alpha\left[1-\tau^{\prime}(z) \dot{z}\right] y e^{-\gamma \tau(z)}-\beta y^{2}=0 .
\end{array}\right.
$$

It is clear that system (2.2) has an equilibrium $E_{0}=(0,0)$. 
Theorem 3.5 System (2.2) has exactly one nontrivial equilibrium $E^{*}=\left(x^{*}, y^{*}\right)$, where $\alpha e^{-\gamma \tau\left(z^{*}\right)}=\alpha e^{-\gamma \tau\left(x^{*}+y^{*}\right)}=\beta y^{*}$.

Proof Due to $\dot{z}=\dot{x}+\dot{y}$, if there is a nontrivial equilibrium $E^{*}=\left(x^{*}, y^{*}\right)$, it must satisfy the following equations:

$$
\left\{\begin{array}{l}
\alpha y-\gamma x-\alpha y e^{-\gamma \tau(z)}=0 \\
\alpha y e^{-\gamma \tau(z)}-\beta y^{2}=0
\end{array}\right.
$$

Adding the two equations of (3.2), we have

$$
x=g(y)=\gamma^{-1}\left(\alpha y-\beta y^{2}\right) .
$$

It is essential that we need $0<y<\alpha \beta^{-1}$ to make sure that $x>0$. According to Theorem 3.3 and $\alpha \beta^{-1} e^{-r \tau_{m}}<\alpha \beta^{-1}$, we will discuss the existence and uniqueness of nontrivial equilibrium in $\Lambda=\left\{y \in \mathbb{R} \mid 0<y<\alpha \beta^{-1} e^{-r \tau_{m}}\right\} \subset \mathbb{R}$. Define $f: \bar{\Lambda} \rightarrow \mathbb{R}$ as a continuous mapping by

$$
f(y)=h(y)-\beta y=\alpha e^{-\gamma \tau(y+g(y))}-\beta y,
$$

where $h(y)=\alpha e^{-\gamma \tau(y+g(y))}$ is a bounded function, namely $\alpha e^{-\gamma \tau_{M}} \leq h(y) \leq \alpha e^{-\gamma \tau_{m}}$.

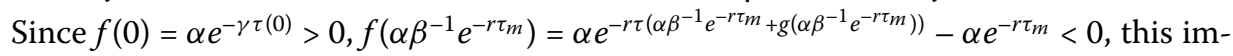
plies that $f(y)$ has at least one positive zero point $y^{*}$.

Note that

$$
\begin{aligned}
f^{\prime}(y)= & -\alpha e^{-\gamma \tau(y+g(y))} \tau^{\prime}(y+g(y))(\gamma+\alpha-2 \beta y)-\beta, \\
f^{\prime}(0)= & -\alpha e^{-\gamma \tau_{m}} \tau^{\prime}(0)(\gamma+\alpha)-\beta<0, \\
f^{\prime \prime}(y)= & \alpha e^{-\gamma \tau(y+g(y))}\left[\tau^{\prime}(y+g(y))\right]^{2}(\gamma+\alpha-2 \beta y)^{2}+2 \alpha \beta e^{-\gamma \tau(y+g(y))} \tau^{\prime}(y+g(y)) \\
& -\frac{\alpha}{\gamma} e^{-\gamma \tau(y+g(y))} \tau^{\prime \prime}(y+g(y))(\gamma+\alpha-2 \beta y)^{2}>0 .
\end{aligned}
$$

Without loss of generality, assume that $f(y)$ has two positive roots and the right positive root is denoted as $y^{*}$. If $f^{\prime}\left(y^{*}\right) \geq 0$, we have $f\left(\alpha \beta^{-1} e^{-r \tau_{m}}\right)>0$ due to $f^{\prime \prime}(y)>0$, giving us a contradiction. Therefore, $f(y)$ has a unique positive root in the interval $\left(0, \alpha \beta^{-1} e^{-r \tau_{m}}\right)$, so system (2.2) has a unique nontrivial equilibrium. The proof is complete.

Remark 3.2 By the proof of Theorem 3.5, we have $f^{\prime}\left(y^{*}\right)<0$, namely

$$
y^{*} \tau^{\prime}\left(z^{*}\right)\left(2 \beta y^{*}-\gamma-\alpha\right)<1
$$

\section{Stability of equilibria}

In this section, we study the linearized stability of the two equilibria $E_{0}$ and $E^{*}$ by linearizing system (2.2). Since the delay is a function depending on the state variables $x$ and $y$, linearizing an equation with state-dependent delay is not completely straightforward. In 1996, Cooke and Huang [33] solved the linearization problem of state-dependent delay 
differential equations by "freezing the delay" at an equilibrium. We use the same method to linearize system (2.2). To do so, let $E^{*}$ be an arbitrary equilibrium and $x=x^{*}+x_{1}$ and $y=y^{*}+y_{1}$. We shall give the details of linearizing the first equation of system (2.2), and linearizing the second could be accomplished similarly. The first equation is linearized as follows:

$$
\begin{aligned}
\dot{x}_{1}= & \alpha\left(y^{*}+y_{1}\right)-\gamma\left(x^{*}+x_{1}\right) \\
& -\alpha\left[1-\tau^{\prime}\left(z^{*}+x_{1}+y_{1}\right)\left(x_{1}^{\prime}+y_{1}^{\prime}\right)\right] y\left(t-\tau\left(z^{*}+x_{1}+y_{1}\right)\right) e^{-\gamma \tau\left(z^{*}+x_{1}+y_{1}\right)} \\
= & -\gamma x_{1}+\alpha y_{1}+\alpha y^{*} \tau^{\prime} z^{*}\left(\gamma+\alpha-2 \beta y^{*}\right) y_{1} e^{-\gamma \tau\left(z^{*}\right)}-\alpha e^{-\gamma \tau\left(z^{*}\right)} y_{1}\left(t-\tau\left(z^{*}\right)\right) \\
= & -\gamma x_{1}+\left[\alpha+\alpha y^{*} \tau^{\prime}\left(z^{*}\right)\left(\gamma+\alpha-2 \beta y^{*}\right) e^{-\gamma \tau\left(z^{*}\right)}\right] y_{1}-\alpha e^{-\gamma \tau\left(z^{*}\right)} y_{1}\left(t-\tau\left(z^{*}\right)\right) .
\end{aligned}
$$

Therefore, the linearized system of (2.2) is

$$
\left\{\begin{array}{l}
\dot{x}_{1}=-\gamma x_{1}+\left[\alpha+\alpha y^{*} \tau^{\prime}\left(z^{*}\right)\left(\gamma+\alpha-2 \beta y^{*}\right) e^{-\gamma \tau\left(z^{*}\right)}\right] y_{1}-\alpha e^{-\gamma \tau\left(z^{*}\right)} y_{1}\left(t-\tau\left(z^{*}\right)\right), \\
\dot{y}_{1}=-\left[\alpha y^{*} \tau^{\prime}\left(z^{*}\right)\left(\gamma+\alpha-2 \beta y^{*}\right) e^{-\gamma \tau\left(z^{*}\right)}+2 \beta y^{*}\right] y_{1}+\alpha e^{-\gamma \tau\left(z^{*}\right)} y_{1}\left(t-\tau\left(z^{*}\right)\right) .
\end{array}\right.
$$

This leads to the following characteristic equation:

$$
(\lambda+\gamma)\left(\lambda+2 \beta y^{*}+\theta^{*}-\alpha e^{-\tau\left(z^{*}\right)(\gamma+\lambda)}\right)=0,
$$

where

$$
\theta^{*}=\alpha y^{*} \tau^{\prime}\left(z^{*}\right)\left(\gamma+\alpha-2 \beta y^{*}\right) e^{-\gamma \tau\left(z^{*}\right)}
$$

\subsection{Linearized stability of the extinction equilibrium $E_{0}$}

For the extinction equilibrium $E_{0}=(0,0),(4.1)$ reduces to

$$
(\lambda+\gamma)\left(\lambda-\alpha e^{-\tau(0)(\gamma+\lambda)}\right)=0 .
$$

Clearly, $\lambda=-\gamma<0$ is one of these eigenvalues. All the other eigenvalues $\lambda$ satisfy the equation $\lambda e^{\tau(0)(\gamma+\lambda)}=\alpha>0$, which always has a real, positive solution. Hence $E_{0}=(0,0)$ is a saddle point. We have the following result.

Theorem 4.1 The extinction equilibrium $E_{0}=(0,0)$ is a saddle point and is unstable.

\subsection{Linearized stability of the nontrivial equilibrium $E^{*}$}

Theorem 4.2 The nontrivial equilibrium $E^{*}=\left(x^{*}, y^{*}\right)$ is locally asymptotically stable.

Proof The characteristic equation

$$
(\lambda+\gamma)\left(\lambda+2 \beta y^{*}+\theta^{*}-\alpha e^{-\tau\left(z^{*}\right)(\gamma+\lambda)}\right)=0,
$$

that is,

$$
(\lambda+\gamma)\left(\lambda-\beta y^{*} e^{-\tau\left(z^{*}\right) \lambda}+\eta\right)=0,
$$


where

$$
\eta=\beta y^{*}\left[2+y^{*} \tau^{\prime}\left(z^{*}\right)\left(\gamma+\alpha-2 \beta y^{*}\right)\right]
$$

Obviously, equation (4.2) has a negative real $\operatorname{root} \lambda=-\gamma$.

Other roots are given by the following equation:

$$
G(\lambda)=\lambda-\beta y^{*} e^{-\tau\left(z^{*}\right) \lambda}+\eta=0 .
$$

By inequality (3.3) of Remark 3.2, we have $G(0)=\eta-\beta y^{*}>0$. Hence, zero is not the root of equation (4.3).

Now, let us prove that equation (4.3) has no purely imaginary roots.

Assume that equation (4.3) has a purely imaginary root $\lambda=i v$, where $v>0$. Substituting it into equation (4.3) and separating the real and the imaginary parts, we obtain

$$
\left\{\begin{array}{l}
v=-\beta y^{*} \sin \left(\tau\left(z^{*}\right) v\right), \\
\eta=\beta y^{*} \cos \left(\tau\left(z^{*}\right) v\right) .
\end{array}\right.
$$

Given $\left(\sin \left(\tau\left(z^{*}\right) v\right)\right)^{2}+\left(\cos \left(\tau\left(z^{*}\right) v\right)\right)^{2}=1$ and inequality (3.3), we have

$$
\begin{aligned}
v^{2} & =\left(\beta y^{*}\right)^{2}-\eta^{2} \\
& =\left(\beta y^{*}\right)^{2}-\left(\beta y^{*}\right)^{2}\left[2+y^{*} \tau^{\prime}\left(z^{*}\right)\left(\gamma+\alpha-2 \beta y^{*}\right)\right]^{2} \\
& =\left(\beta y^{*}\right)^{2}\left[1-\left(2+y^{*} \tau^{\prime}\left(z^{*}\right)\left(\gamma+\alpha-2 \beta y^{*}\right)\right)^{2}\right] \\
& <0,
\end{aligned}
$$

which is a contradiction. Therefore, equation (4.3) has no purely imaginary roots, and each root of the characteristic equation has a negative real part. The proof is complete.

\section{Permanence}

In this section, we will discuss the permanence of system (2.2) by using the method of $[5,28]$, and obtain explicit bounds for the eventual behaviors of $x(t)$ and $y(t)$, which are independent of the initial conditions.

Definition 5.1 System (2.2) is said to be permanent if there exist positive constants $m, n$, $M$, and $N$ such that every positive solution $(x(t), y(t))$ of system (2.2) satisfies

$$
\begin{gathered}
m \leq \liminf _{t \rightarrow \infty} x(t) \leq \limsup _{t \rightarrow \infty} x(t) \leq M, \\
n \leq \liminf _{t \rightarrow \infty} y(t) \leq \limsup _{t \rightarrow \infty} y(t) \leq N .
\end{gathered}
$$

In order to obtain explicit bounds for the eventual behavior of $y(t)$, we first investigate the upper and lower bounds of $\lim _{t \rightarrow \infty} y(t)$ in the case where $y(t)$ is eventually monotone.

Theorem 5.1 Suppose that $y(t)$ is eventually monotone. Then

$$
\alpha \beta^{-1} e^{-\gamma \tau_{M}} \leq \lim _{t \rightarrow \infty} y(t) \leq \alpha \beta^{-1} e^{-\gamma \tau_{m}} .
$$


Proof Since $y(t)$ is eventually monotone and bounded, there exists a $0 \leq \bar{y}<\infty$ such that $\lim _{t \rightarrow \infty} y(t)=\bar{y}$ and $\lim _{t \rightarrow \infty} \dot{y}(t)=0$. Because the zero solution is unstable by Theorem 4.1, we obtain $\bar{y} \neq 0$, namely $0<\bar{y}<\infty$. For the boundedness of $z(t)$ and $\dot{z}(t)=-\gamma z(t)+(\alpha+$ $\gamma) y(t)-\beta y^{2}(t)$, according to the Barbǎlat lemma [34], we have $\lim _{t \rightarrow \infty} \dot{z}(t)=0$. Thus, from system (2.2), taking the limit superior as $t \rightarrow \infty$, we get that

$$
0 \leq \bar{y}\left[\alpha e^{-\gamma \liminf _{t \rightarrow \infty} \tau(z(t))}-\beta \bar{y}\right] .
$$

Hence $\bar{y} \leq \alpha \beta^{-1} e^{-\gamma \liminf t \rightarrow \infty \tau(z(t))} \leq \alpha \beta^{-1} e^{-\gamma \tau_{m}}$ by combining with the hypotheses (A2).

Similarly, from system (2.2), taking the limit infimum as $t \rightarrow \infty$, we obtain that

$$
0 \geq \bar{y}\left[\alpha e^{-\gamma \lim \sup _{t \rightarrow \infty} \tau(z(t))}-\beta \bar{y}\right] .
$$

Thus $\bar{y} \geq \alpha \beta^{-1} e^{-\gamma \limsup t \rightarrow \infty} \tau(z(t)) \geq \alpha \beta^{-1} e^{-\gamma \tau_{M}}$ by the hypotheses (A2). The proof is complete.

Now let us show explicit bounds, independent of initial conditions, for $y(t)$.

Theorem 5.2 There exist positive constants $n$ and $N$ such that, for any positive solution $(x(t), y(t))$ of model (2.2),

$$
n \leq \liminf _{t \rightarrow \infty} y(t) \leq \limsup _{t \rightarrow \infty} y(t) \leq N
$$

where $n=\alpha \beta^{-1} e^{-\gamma \tau_{M}}, N=\alpha \beta^{-1} e^{-\gamma \tau_{m}}$.

Proof Our proof is split into two cases.

In the first case, $y(t)$ is eventually monotonic. According to Theorem 5.1 and the definitions of superior and inferior limits, the claim is true.

Secondly, we consider an oscillatory $y(t)$. We only show that $\lim _{\sup _{t \rightarrow \infty}} y(t) \leq \alpha \beta^{-1} \times$ $e^{-\gamma \tau_{m}}$, because the other inequality can be obtained analogously. Define the sequence $\left\{t_{m}\right\}$ as those times for which $y(t)$ obtains its local maximum, i.e., $\dot{y}\left(t_{m}\right)=0, \ddot{y}\left(t_{m}\right)<0$.

Let $\widehat{y}=\lim \sup _{m \rightarrow \infty} y\left(t_{m}\right)$. Then $\hat{y}=\limsup _{t \rightarrow \infty} y(t)$. If $\widehat{y} \leq \alpha \beta^{-1} e^{-\gamma \tau_{m}}$, we are done. Therefore, we assume that

$$
\widehat{y}>\alpha \beta^{-1} e^{-\gamma \tau_{m}} .
$$

Then $0=\dot{y}\left(t_{m}\right)=\alpha\left[1-\tau^{\prime}\left(z_{m}\right) \dot{z}\left(t_{m}\right)\right] y\left(t-\tau\left(z_{m}\right)\right) e^{-\gamma \tau\left(z\left(t_{m}\right)\right)}-\beta y^{2}\left(t_{m}\right)$, where $z_{m}=z\left(t_{m}\right)=$ $x\left(t_{m}\right)+y\left(t_{m}\right)$.

Now let us choose a subsequence of $\left\{t_{m}\right\}$, labeled as $\left\{t_{m_{1}}\right\}$, so that $\lim _{m_{1} \rightarrow \infty} y\left(t_{m_{1}}\right)=\widehat{y}$ and $\lim \sup _{m_{1} \rightarrow \infty} z\left(t_{m_{1}}\right)=\widehat{z}$.

We then choose a subsequence of $\left\{t_{m_{1}}\right\}$, labeled as $\left\{t_{m_{2}}\right\}$, so that $\lim _{m_{2} \rightarrow \infty} z\left(t_{m_{2}}\right)=\widehat{z}$ and $\lim \sup _{m_{2} \rightarrow \infty} y\left(t_{m_{2}}-\tau\left(z_{m_{2}}\right)\right)=\tilde{y}$.

Finally, we choose a subsequence of $\left\{t_{m_{2}}\right\}$, labeled as $\left\{t_{m_{3}}\right\}$, so that $\lim _{m_{3} \rightarrow \infty} y\left(t_{m_{3}}-\right.$ $\left.\tau\left(z_{m_{3}}\right)\right)=\tilde{y}$. 
Then from (2.2) and (5.1), taking the limit as $m_{1} \rightarrow \infty, m_{2} \rightarrow \infty, m_{3} \rightarrow \infty$,

$$
\begin{aligned}
0 & =\alpha\left[1-\tau^{\prime}(\widehat{z}) \dot{\bar{z}}\right] \tilde{y} e^{-\gamma \tau(\hat{z})}-\beta \widehat{y}^{2} \\
& <\alpha\left[1-\tau^{\prime}(\widehat{z}) \dot{\bar{z}}\right] e^{-\gamma \tau(\bar{z})}(\widetilde{y}-\widehat{y}) .
\end{aligned}
$$

If $\tilde{y} \leq \widehat{y}$, we have a contradiction. Therefore, assuming $\tilde{y}>\widehat{y}$, we can choose a value $t_{n}$ for $m, m_{1}, m_{2}, m_{3}$ such that $\dot{y}\left(t_{n}\right)=0, \ddot{y}\left(t_{n}\right)<0$, and $\limsup _{n \rightarrow \infty} y\left(t_{n}\right) \geq \tilde{y}>\widehat{y}$. However, this contradicts the definition of $\tilde{y}$, therefore $\tilde{y}>\hat{y}$ cannot be true, which eliminates the last possibility. The proof is complete.

Now let us give estimates for $x$ by using the estimates obtained in Theorem 5.1. It should be noted that we can find a $T(\epsilon)>0$ so large that

$$
\alpha \beta^{-1} e^{-\gamma \tau_{M}}-\epsilon \leq y(t) \leq \alpha \beta^{-1} e^{-\gamma \tau_{m}}+\epsilon
$$

for any given $\epsilon>0$ whenever $t \geq T$. Clearly, inequality (5.2) is also valid for those $t \geq$ $T+\tau_{M}$.

Theorem 5.3 There exist positive constants $m$ and $M$ such that, for any positive solution $(x(t), y(t))$ of model (2.2),

$$
m \leq \liminf _{t \rightarrow \infty} x(t) \leq \limsup _{t \rightarrow \infty} x(t) \leq M
$$

where $m=\alpha^{2} \gamma^{-1} \beta^{-1} e^{-\gamma \tau_{M}}\left(1-e^{-\gamma \tau_{m}}\right), M=\alpha^{2} \gamma^{-1} \beta^{-1} e^{-\gamma \tau_{m}}\left(1-e^{-\gamma \tau_{M}}\right)$.

Proof Since the first equation of system (2.2) can be written in the integral equation form and utilizing inequality (5.2), we have for $t \geq T+\tau_{M}$,

$$
\begin{aligned}
x(t) & =\int_{t-\tau(z(t))}^{t} \alpha y(\xi) e^{-\gamma(t-\xi)} d \xi \\
& \leq \int_{t-\tau(z(t))}^{t} \alpha\left(\alpha \beta^{-1} e^{-\gamma \tau_{m}}+\epsilon\right) e^{-\gamma(t-\xi)} d \xi \\
& =\alpha\left(\alpha \beta^{-1} e^{-\gamma \tau_{m}}+\epsilon\right) \int_{t-\tau(z(t))}^{t} e^{-\gamma(t-\xi)} d \xi \\
& =\alpha \gamma^{-1}\left(\alpha \beta^{-1} e^{-\gamma \tau_{m}}+\epsilon\right)\left[1-e^{-\gamma \tau(z(t))}\right],
\end{aligned}
$$

where $\epsilon>0$ is arbitrary. Thus we have

$$
\begin{aligned}
\limsup _{t \rightarrow \infty} x(t) & \leq \limsup _{t \rightarrow \infty} \alpha \gamma^{-1}\left(\alpha \beta^{-1} e^{-\gamma \tau_{m}}+\epsilon\right)\left[1-e^{-\gamma \tau(z(t))}\right] \\
& \leq \alpha \gamma^{-1}\left(\alpha \beta^{-1} e^{-\gamma \tau_{m}}+\epsilon\right)\left(1-e^{-\gamma \tau_{M}}\right) \\
& =\alpha^{2} \gamma^{-1} \beta^{-1} e^{-\gamma \tau_{m}}\left(1-e^{-\gamma \tau_{M}}\right)+\epsilon \alpha \gamma^{-1}\left(1-e^{-\gamma \tau_{M}}\right) .
\end{aligned}
$$

Since $\epsilon$ is arbitrary, we get an upper bound on $x(t)$. 
Similarly, we have for $t \geq T+\tau_{M}$ that

$$
\begin{aligned}
x(t) & \geq \int_{t-\tau(z(t))}^{t} \alpha\left(\alpha \beta^{-1} e^{-\gamma \tau_{M}}-\epsilon\right) e^{-\gamma(t-\xi)} d \xi \\
& =\alpha \gamma^{-1}\left(\alpha \beta^{-1} e^{-\gamma \tau_{M}}-\epsilon\right)\left[1-e^{-\gamma \tau(z(t))}\right],
\end{aligned}
$$

where $\epsilon>0$ is arbitrary. Then we obtain

$$
\begin{aligned}
\liminf _{t \rightarrow \infty} x(t) & \geq \liminf _{t \rightarrow \infty} \alpha \gamma^{-1}\left(\alpha \beta^{-1} e^{-\gamma \tau_{M}}-\epsilon\right)\left[1-e^{-\gamma \tau(z(t))}\right] \\
& \geq \alpha \gamma^{-1}\left(\alpha \beta^{-1} e^{-\gamma \tau_{M}}-\epsilon\right)\left(1-e^{-\gamma \tau_{m}}\right) \\
& =\alpha^{2} \gamma^{-1} \beta^{-1} e^{-\gamma \tau_{M}}\left(1-e^{-\gamma \tau_{m}}\right)-\epsilon \alpha \gamma^{-1}\left(1-e^{-\gamma \tau_{m}}\right) .
\end{aligned}
$$

Therefore, we get a lower bound on $x(t)$ by the arbitrariness of $\epsilon$. The proof is complete.

Remark 5.1 In Theorem 5.6 of [14] and Theorem 5.4 of [28], one requires that $\tau_{M}<2 \tau_{m}$ for the lower bound to be positive. In fact, according to Theorem 5.3, we see that this condition is not necessary.

\section{Conclusions and discussions}

In this paper, based on the biological observations that during World War II the maturation time of seals and whales was not a fixed value, but depended on the total population (immature and mature), starting with an age-structured model (2.1), we formulated and analyzed a novel stage-structured model with state-dependent maturity delay.

Compared with the previous state-dependent delay equations (e.g., [5, 26, 28, 30]), model (2.2) is not directly changing the constant delay $\tau$ into a state-dependent delay $\tau(z(t))$ but was obtained by reducing the age-structured population model, which has the correction term $1-\tau^{\prime}(z(t)) \dot{z}(t)$. Biologically speaking, model (2.2) is appropriate in terms of population modeling. On the one hand, with the state-dependent maturity delay, the changes in the number of mature individuals depend on reproduction and death and the changing definition of maturity, which is in line with the correction term $1-\tau^{\prime}(z(t)) \dot{z}(t)$. On the other hand, we can represent $x(t)$ in an integral form by some biological inductions, namely

$$
x(t)=\int_{t-\tau(z(t))}^{t} \alpha y(\xi) e^{-\gamma(t-\xi)} d \xi
$$

Taking the derivatives of $x(t)$, we obtain the first equation of model (2.2).

From a biological point of view, we show that $t-\tau(z(t))$ should be a strictly increasing function of $t$ without any conditions and the derivative with respect to time of the statedependent maturity delay $\tau(z(t))$ is strictly less than one. In addition, it is biologically reasonable for the assumption of the delay $\tau(z(t))$. The biological phenomenon mentioned above, a non-decreasing delay, implies that a larger population leads to a longer developmental duration, and it makes clear the stabilizing effect [15, 35].

Mathematically compared with [5, 28], first of all, the positivity and boundedness of solutions are discussed, which do not need the stringent condition on $\tau^{\prime}(z(t))$ to ensure 
the positivity of $x$. Then the existence and uniqueness of all equilibria are formulated, which are different from the result of [5]. Besides, the nontrivial equilibrium $E^{*}$ is always linearly stable without auxiliary conditions. Finally, we discuss some global properties of the solutions of our model (2.2) and obtain explicit bounds for the eventual behaviors of $x(t)$ and $y(t)$, which do not need the condition $\tau_{M}<2 \tau_{m}$ for the lower bound of $x(t)$.

Further research in this direction may consider more realistic complex models, for example, a multi-stage structure population model with state-dependent delays and a multipopulation model with state-dependent delays.

\section{Funding}

This work was supported by the National Natural Science Foundation of China (11671327) and the Fundamental Research Funds for the Central Universities (XDJK2018D024).

\section{Competing interests}

The authors declare that they have no competing interests.

\section{Authors' contributions}

The authors contributed equally to the writing of this paper. The authors read and approved the final manuscript.

\section{Author details}

${ }^{1}$ Key Laboratory of Eco-environments in Three Gorges Reservoir Region (Ministry of Education), School of Mathematics and Statistics, Southwest University, Chongqing, China. ${ }^{2}$ School of Mathematics and Statistics, Guangxi Teachers Education University, Nanning, China.

\section{Publisher's Note}

Springer Nature remains neutral with regard to jurisdictional claims in published maps and institutional affiliations.

Received: 5 September 2018 Accepted: 28 September 2018 Published online: 10 October 2018

\section{References}

1. Landahl, H.D., Hansen, B.D.: A three stage population model with cannibalism. Bull. Math. Biol. 37(1), 11-17 (1975)

2. Wangersky, P.J., Cunningham, W.J.: On time lags in equations of growth. Proc. Natl. Acad. Sci. USA 42(9), 699-702 (1956)

3. Wangersky, P.J., Cunningham, W.J.: Time lag in prey-predator population models. Ecology 38(1), 136-139 (1957)

4. Huang, C., Qiao, Y., Huang, L., Agarwal, R.P.: Dynamical behaviors of a food-chain model with stage structure and time delays. Adv. Differ. Equ. 2018(1), 186 (2018)

5. Aiello, W.G., Freedman, H.I., Wu, J.: Analysis of a model representing stage-structured population growth with state-dependent time delay. SIAM J. Appl. Math. 52(3), 855-869 (1992)

6. Barclay, H.J., van den Driessche, P.: A model for a species with two life history stages and added mortality. Ecol. Model. 11(3), 157-166 (1980)

7. Gurney, W.S.C., Nisbet, R.M., Lawton, J.H.: The systematic formulation of tractable single-species population models incorporating age structure. J. Anim. Ecol. 52(2), 479-495 (1983)

8. Lou, Y., Zhao, X.-Q.: A theoretical approach to understanding population dynamics with seasonal developmental durations. J. Nonlinear Sci. 27(2), 573-603 (2017)

9. Rezounenko, A.V.: Partial differential equations with discrete and distributed state-dependent delays. J. Math. Anal. Appl. 326(2), 1031-1045 (2007)

10. Tognetti, K.: The two stage stochastic population model. Math. Biosci. 25(3), 195-204 (1975)

11. Huang, C., Yang, Z., Yi, T., Zou, X.: On the basins of attraction for a class of delay differential equations with non-monotone bistable nonlinearities. J. Differ. Equ. 256(7), 2101-2114 (2014)

12. Gurney, W.S.C., Blythe, S.P., Nisbet, R.M.: Nicholson's blowflies revisited. Nature 287(5777), 17-21 (1980)

13. Nicholson, A.J.: Compensatory reactions of populations to stresses, and their evolutionary significance. Aust. J. Zool. 2(1), 1-8 (1954)

14. Aiello, W.G., Freedman, H.I.: A time-delay model of single-species growth with stage structure. Math. Biosci. 101(2), 139-153 (1990)

15. Gambell, R.: Birds and mammals - Antarctic whales. In: Bonner, W., Walton, D. (eds.) Antarctica. Pergamon Press, New York (1985)

16. Guo, S., Wu, J.: Bifurcation Theory of Functional Differential Equations, pp. 81-88. Springer, New York (2013)

17. Hou, A., Guo, S.: Stability and Hopf bifurcation in van der Pol oscillators with state-dependent delayed feedback. Nonlinear Dyn. 79(4), 2407-2419 (2015)

18. Hou, A., Guo, S.: Stability and bifurcation in a state-dependent delayed predator-prey system. Int. J. Bifurc. Chaos Appl. Sci. Eng. 26(4), 1650060 (2016)

19. LV, Y., Pei, Y., Yuan, R.: Modeling and analysis of a predator-prey model with state-dependent delay. Int. J. Biomath $11(2), 1850026(2018)$

20. Al-Omari, J.F.M., Gourley, S.A.: Stability and traveling fronts in Lotka-Volterra competition models with stage structure. SIAM J. Appl. Math. 63(6), 2063-2086 (2003) 
21. Chen, F., Sun, D., Shi, J.: Periodicity in a food-limited population model with toxicants and state dependent delays. J. Math. Anal. Appl. 288(1), 136-146 (2003)

22. Hu, Q., Zhao, X.-Q.: Global dynamics of a state-dependent delay model with unimodal feedback. J. Math. Anal. Appl. 399(1), 133-146 (2013)

23. Kloosterman, M., Campbell, S.A., Poulin, F.J.: An NPZ model with state-dependent delay due to size-structure in juvenile zooplankton. SIAM J. Appl. Math. 76(2), 551-577 (2016)

24. Zaghrout, A.A.S., Attalah, S.H.: Analysis of a model of stage-structured population dynamics growth with time state-dependent time delay. Appl. Math. Comput. 77(2-3), 185-194 (1996)

25. Zhang, L., Guo, S.: Slowly oscillating periodic solutions for the Nicholson's blowflies equation with state-dependent delay. Math. Methods Appl. Sci. 145(11), 4893-4903 (2017)

26. Al-Omari, J.F.M., Gourley, S.A.: Dynamics of a stage-structured population model incorporating a state-dependent maturation delay. Nonlinear Anal., Real World Appl. 6(1), 13-33 (2004)

27. LV, Y., Yuan, R.: Global stability and wavefronts in a cooperation model with state-dependent time delay. J. Math. Anal. Appl. 415(2), 543-573 (2014)

28. Li, S., Guo, S.: Dynamics of a two-species stage-structured model incorporating state-dependent maturation delays. Discrete Contin. Dyn. Syst., Ser. B 22(4), 1393-1423 (2017)

29. Macdonald, N.: Biological Delay Systems: Linear Stability Theory. Cambridge University Press, New York (1989)

30. Barbarossa, M.V., Hadeler, K.P., Kuttler, C.: State-dependent neutral delay equations from population dynamics. J. Math. Biol. 69(4), 1027-1056 (2014)

31. Cushing, J.M.: An Introduction to Structured Population Dynamics, pp. 171-174. SIAM, Philadelphia (1998)

32. Webb, G.F.: Theory of Nonlinear Age-Dependent Population Dynamics. Dekker, New York (1985)

33. Cooke, K.L., Huang, W.Z.: On the problem of linearization for state-dependent delay differential equations. Proc. Am. Math. Soc. 124(5), 1417-1426 (1996)

34. Gopalsamy, K.: Stability and Oscillations in Delay Differential Equations of Population Dynamics. Springer, Netherlands (1992)

35. Trippel, E.A.: Age at maturity as a stress indicator in fisheries. Bioscience 45(11), 759-771 (1995)

\section{Submit your manuscript to a SpringerOpen ${ }^{\circ}$ journal and benefit from:}

- Convenient online submission

Rigorous peer review

Open access: articles freely available online

- High visibility within the field

- Retaining the copyright to your article

Submit your next manuscript at $\gg$ springeropen.com 